\section{Surface Texture Metrological Characteristics}

\author{
Han Haitjema ${ }^{1}$ and Richard Leach ${ }^{2}$ \\ ${ }^{1} \mathrm{KU}$ Leuven, Department of Mechanical \\ Engineering, Leuven, Belgium \\ ${ }^{2}$ Manufacturing Metrology Team, Faculty of \\ Engineering, University of Nottingham, \\ Nottingham, UK
}

\section{Synonym}

Surface texture measuring instrument specifications

\section{Definition}

The surface texture metrological characteristics establish a common calibration framework for surface topography measuring instruments. A metrological characteristic is defined in ISO specification standards as a "Characteristic of measuring equipment, which may influence the results of measurements."

\section{Theory and Application}

\section{Introduction}

In general, surface topography measuring instruments measure the height $z$ of a surface, relative to a nominally horizontal $x-y$ plane. For instruments that scan in the horizontal plane, the $x$-axis is the tracing axis, and the $y$-axis is the stepping axis. For optical measurements where the $x-y$ plane is projected on a camera, the pixels correspond to the $(x, y)$ coordinates, and the $z$ coordinates are obtained by some operation, e.g., from a stack of images while the surface is moved in the $z$-direction.

The metrological characteristics of surface topography measuring instruments are given in the literature (Leach 2011; Leach et al. 2015) and in several ISO specification standards. Several standards (ISO 25178-601 2010; ISO 25178602 2010; ISO 25178-603 2013; ISO 25178-604 2013; ISO 25178-605 2014; ISO 25178-606 2015) have been developed to define terms and metrological characteristics for individual measurement methods. At the time of writing, the common aspects of the methods are being summarized into one standard: ISO DIS 25178-600 (2018). The metrological characteristics for surface topography measuring instruments are summarized in ISO DIS 25178-600 and presented in Table 1. 
In the following sections, the metrological characteristics and their determination (calibration) will be briefly explained.

\section{Amplification Coefficient $\boldsymbol{\alpha}_{x}, \boldsymbol{\alpha}_{y}, \boldsymbol{\alpha}_{z}$}

The amplification coefficient is the slope of the linear regression curve obtained from the response function. Amplification coefficients apply to the $x$, $y$, and $z$ coordinates. The ideal response is a straight line with a slope equal to 1 , which means that the values of the measurand are equal to the values of the input quantities. The amplification coefficient quantity may also be termed "scaling factor."

\section{Linearity Deviation $I_{x}, I_{y}, I_{z}$}

The linearity deviation is the maximum local difference between the curve from which the amplification coefficient is derived and the response function.

For the linearity and amplification in the $z$-direction, measurement of step heights is the default method for determination of the amplification coefficient $\alpha_{\mathrm{z}}$ and linearity deviation $l_{\mathrm{z}}$. The measured depth is compared with the calibrated depth of the material measure (Giusca et al. $2012 b$ ). The major drawback of this type of artifact is that they only reproduce a single value, usually much smaller than the instrument's measurement range; consequently, it is often not possible to establish the amplification coefficient to within a useful uncertainty. A solution to this drawback is to use multiple step height artifacts of different values (Giusca et al. 2012a) or a material measure with multiple heights of different values (Brand et al. 2006). Alternative methods may use interferometric displacement sensors, either directly (de Groot and Beverage 2015) or indirectly by a separate calibration device, a so-called vibration table (Haitjema and Morel 2005a; Koops et al. 2015). An efficient way of checking the $z$-linearity deviation $l_{z}$ in a limited range is by measuring a tilted optical flat (see ISO 12179 2000).

For the $x-y$ calibration, the measurement of a calibrated line scale, calibrated sinusoidal profile, or a calibrated cross-grating is most appropriate (Giusca et al. 2012a). A virtual lateral calibration
Surface Texture Metrological Characteristics, Table 1 Metrological characteristics for surface topography measuring instruments

\begin{tabular}{l|l|l}
\hline $\begin{array}{l}\text { Metrological } \\
\text { characteristic }\end{array}$ & Symbol & $\begin{array}{l}\text { Main } \\
\text { potential } \\
\text { error along }\end{array}$ \\
\hline Amplification coefficient & $\alpha_{x}, \alpha_{y}, \alpha_{z}$ & $x, y, z$ \\
\hline Linearity deviation & $l_{x}, l_{y}, l_{z}$ & $x, y, z$ \\
\hline Flatness deviation & $z_{\mathrm{FLT}}$ & $z$ \\
\hline Measurement noise & $N_{\mathrm{M}}$ & $z$ \\
\hline $\begin{array}{l}\text { Topographic spatial } \\
\text { resolution }\end{array}$ & $W_{\mathrm{R}}$ & $z, x, y$ \\
\hline$x-y$ mapping deviation & $\Delta_{x}(x, y)$, & $x, y$ \\
\hline Topography fidelity & $\Delta_{y}(x, y)$ & $T_{\mathrm{FI}}$ \\
\hline
\end{tabular}

standard has been developed where a specific feature is given a known lateral displacement while it is measured or scanned by the topography measuring instrument (Koops et al. 2016).

Figure 1 shows an example of how quantities $\alpha_{z}$ and $l_{z}$ can be derived from a response curve that is obtained by calibration.

\section{Flatness Deviation $z_{\mathrm{FLT}}$}

The flatness deviation is the deviation of the measured topography of an ideally flat object from a plane. Flatness deviation can be caused by residual flatness of an imperfect areal reference or by imperfection in the optical setup of an instrument.

An effective way of determining the flatness deviation is the measurement of an optical flat. Commonly, the flatness deviation of optical flats is in the nanometer region for millimeter square regions and can be negligible compared to the flatness deviation of the instrument being calibrated. The influence of the used optical flat can be reduced further by taking a measurement at various locations on the optical flat and averaging the result for every point (pixel) of the measurement (Evans and Tyler Estler 1996; Giusca et al. 2014; Creath and Wyant 1990). Further reduction of the influence of the used flat surface can be achieved by considering the randomness of the Fourier components of the surface (Haitjema and Morel 2005b). 


\section{Surface Texture \\ Metrological \\ Characteristics,}

Fig. 1 Example of a

response curve and

derivation of amplification

factor and linearity

deviation

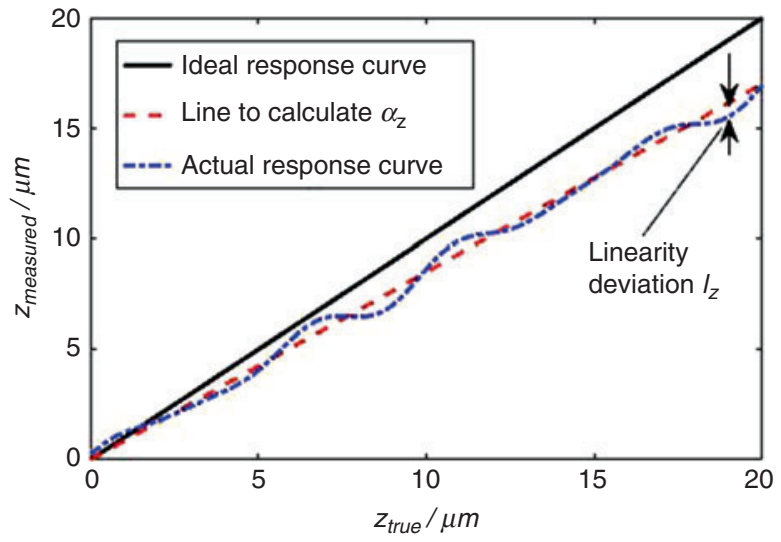

- Stylus tip radius rTIP in case of a mechanical measurement

Measurement noise is the noise added to the output signal occurring during the normal use of the instrument. Measurement noise includes the instrument noise as well as components arising from the environment (thermal, vibration, air turbulence) and other sources. The instrument noise may be due to electronic noise, such as that arising in amplifiers, or optical noise, such as that arising from stray light or from fluctuations in the intensity of the used light source. The noise is commonly specified as a standard deviation in the $z$ coordinates, possibly with a specified filtering applied.

Commonly, only the measurement noise in the $z$-direction is relevant and is determined. An effective method to determine the measurement noise is by repeating the measurement and determining the standard deviation in every coordinate (Haitjema and Morel 2005b; Giusca et al. 2014).

\section{Topographic Spatial Resolution $W_{R}$}

The topographic spatial resolution is the metrological characteristic describing the ability of a surface topography measuring instrument to distinguish closely spaced surface features. Several parameters and functions may be used to quantify the topographic spatial resolution, depending on the application and the method of measurement. These include:

- Lateral period limit DLIM, that is, the spatial period of a sinusoidal profile at which the height response falls to $50 \%$
- Lateral resolution $\mathrm{Rl}$, that is, the smallest distance between two features which can be measured

- Width limit for full height transmission Wl, that is, the width of the narrowest rectangular groove whose step height is measured within a given tolerance

- Small-scale fidelity limit TFIL, that is, smallest lateral surface feature for which the reported topography parameters deviate from accepted values by less than a specified amount, e.g., $10 \%$

- Rayleigh criterion, that is, the quantity characterizing the optical lateral resolution given by the separation of two point sources at which the first diffraction minimum of the intensity image of one point source coincides with the maximum of the other

- Sparrow criterion, that is, the quantity characterizing the optical lateral resolution given by the separation of two point sources at which the second derivative of the intensity distribution vanishes between the two imaged points

The latter two parameters are useful for characterizing the instrument response to features with heights much less than the used effective light wavelength for optical surface topography measuring instruments.

For optical instruments, it is common to specify the topographical spatial resolution based on the theoretically achievable lateral resolution 
using the numerical aperture of the used objective. However, based on the measurement principle and evaluation, the lateral resolution can be much worse in practice (Giusca and Leach 2013; de Groot et al. 2012). The measurement/calibration of the topographic spatial resolution can be carried out using material measures, such as starshaped (Giusca and Leach 2013; Xu et al. 2012) or chirped artifacts characterized by uniform amplitude (Krüger-Sehm et al. 2007) or with varying amplitudes (Fujii et al. 2011).

For mechanical measurements, the stylus tip radius is an appropriate measure, provided the stylus follows the surface faithfully.

\section{$x-y$ Mapping Deviation $\Delta_{x}(x, y), \Delta_{y}(x, y)$}

The $x-y$ mapping deviation is generally displayed as a gridded image of $x$ - and $y$-deviations of actual coordinate positions on a surface from their nominal positions. The mapping deviations may be used to calculate the $x$ - and $y$-amplification and linearity deviations and the $x-y$ axis perpendicularity.

The $x-y$ mapping is most conveniently carried by the use of a calibrated grid (Giusca et al. 2012a).

\section{Topography Fidelity $T_{\mathrm{FI}}$}

Topographic fidelity is the closeness of agreement between a measured surface profile or measured topography and reference values whose uncertainties are insignificant by comparison.

A material measure may be used for the determination of the topography fidelity. The shape of the material measure should be close to the shape of the measurand. To quantify the topography fidelity, a mathematical model or calibrated values of the topography represent the shape of the calibrated material measure. An example structure is a square-profile grating having a depth and periodicity consistent with the intended measured surface structure.

\section{Additional Characteristics}

Other metrological characteristics are in use that are not currently in the ISO 25178-600. A few are mentioned here for completeness and reference.
Maximum Measurable Local Slope

The maximum measurable local slope is the greatest local slope of a surface feature that can be assessed by the measurement system. The maximum measurable slope is an important limitation to be specified for a surface topography measurement instrument. However, calibration of this parameter is rarely useful. For optical systems, the maximum measurable local slope is commonly considered as dependent of the numerical aperture of the used objective, and for mechanical probing systems, it depends on both the probe tip radius and the cone angle. However, this quantity depends on the definition of "local" (e.g., a small local roughness on a tilted area of a specimen); therefore, it is difficult to give a generally valid specification.

\section{RMS Repeatability}

The RMS repeatability is the ability of an instrument to reproduce the "RMS" of a surface, where with "RMS" commonly the $S q$ parameter is intended. The problem with this quantity is that a smaller value does not necessarily imply a "better" instrument; e.g., a high but constant measurement noise may result in a very low (good) RMS repeatability. A critical review of this and some more commonly used specification values (e.g., "vertical resolution") was given by de Groot (2017).

\section{Conclusions and Further Requirements}

At present, instrument manufacturers use a wide range of specifications. The general adoption of the ISO standards as mentioned, and specification of the surface texture metrological characteristics as listed above, would mean a major step forward to clarify and compare the various instruments' performances. From a user's perspective, calibration of the metrological characteristics may be the starting point of an uncertainty assessment of measurements taken by an instrument; however, for a complete understanding and uncertainty estimation for specific measurements, additional information may be needed (Haitjema 2015). Also, a sound and generally applicable topography fidelity assessment method is still to be developed. 


\section{Cross-References}

\author{
- Calibration \\ - Flatness \\ - Roughness \\ - Surface Parameter \\ - Surface Texture \\ - Topography \\ - Traceability
}

\section{References}

Brand U, Schädelnach H, Schödel R, Feist C, Hinzmann G (2006) New depth-setting standards with grooves up to $5 \mathrm{~mm}$ depth. In: Proceedings of 6 th international EUSPEN conference, Baden, 2006, p 438

Creath K, Wyant JC (1990) Absolute measurement of surface roughness. Appl Opt 29(26):3823-3826

de Groot PJ (2017) The meaning and measure of vertical resolution in optical surface topography measurement. Appl Sci 7(1)., art. no. 54

de Groot P, Beverage J (2015) Calibration of the amplification coefficient in interference microscopy by means of a wavelength standard. Proc SPIE 9526:10-11

de Groot P, Colonna de Lega X, Sykorda DM, Deck L (2012) The meaning and measure of lateral resolution for surface profiling interferometers. Opt Photonics News 23(4):10-13

Evans CJ, Tyler Estler W (1996) Self-calibration: reversal, redundancy, error separation, and 'absolute testing'. Ann CIRP 45(2):617-634

Fujii A, Suzuki H, Yanagi K (2011) Development of measurement standards for verifying functional performance of surface texture measuring instruments. J Phys Conf Ser 311:012009

Giusca CL, Leach RK (2013) Calibration of the scales of areal surface topography measuring instruments: part 3 - resolution. Meas Sci Technol 24:105010

Giusca CL, Leach RK, Helery F (2012a) Calibration of the scales of areal surface topography measuring instruments: part 2 - amplification coefficient, linearity and squareness. Meas Sci Technol 23:065005

Giusca CL, Leach RK, Helery F, Gutauskas T, Nimishakavi L (2012b) Calibration of the scales of areal surface topography measuring instruments: part 1 - measurement noise and residual flatness. Meas Sci Technol 23:035008

Giusca CL, Claverley JD, Sun W, Leach RK, Helmli F, Chavigner MPJ (2014) Practical estimation of measurement noise and flatness deviation on focus variation microscopes. Ann CIRP 63(1):545-548

Haitjema H (2015) Uncertainty in measurement of surface topography. Surf Topography Metrol Prop 3(3)., art. no. 035004
Haitjema H, Morel MAA (2005a) Accurate roughness measurements by laser interferometer calibration, VFM-uncertainty calculations and noise reduction. Proc SPIE 58790I:1-7

Haitjema H, Morel MAA (2005b) Noise bias removal in profile measurements. Measurement 38(1):21-29

ISO 12179 (2000) Geometrical product specifications (GPS) - surface texture: profile method - calibration of contact (stylus) instruments. ISO, Geneva

ISO 25178-601 (2010) Geometrical product specifications (GPS) - surface texture: areal - part 601: nominal characteristics of contact (stylus) instruments. ISO, Geneva

ISO 25178-602 (2010) Geometrical product specifications (GPS) - surface texture: areal - part 602: nominal characteristics of non-contact (confocal chromatic probe) instruments. ISO, Geneva

ISO 25178-603 (2013) Geometrical product specifications (GPS) - surface texture: areal - part 603: nominal characteristics of non-contact (phase-shifting interferometric microscopy) instruments. ISO, Geneva

ISO 25178-604 (2013) Geometrical product specifications (GPS) - surface texture: areal - part 604: nominal characteristics of non-contact (coherence scanning interferometry) instruments. ISO, Geneva

ISO 25178-605 (2014) Geometrical product specifications (GPS) - surface texture: areal - part 605: nominal characteristics of non-contact (point autofocus probe) instruments. ISO, Geneva

ISO 25178-606 (2015) Geometrical product specifications (GPS) - surface texture: areal - part 606: nominal characteristics of non-contact (focus variation) instruments. ISO, Geneva

ISO-DIS 25178-600 (2018) Geometrical product specifications (GPS) - surface texture: areal - part 600: metrological characteristics for areal-topography measuring methods. ISO, Geneva

Koops R, Van Veghel M, Van De Nes A (2015) A dedicated calibration standard for nanoscale areal surface texture measurements. Microelectron Eng 141:250-255

Koops R, Van Veghel M, Van De Nes A (2016) A virtual lateral standard for AFM calibration. Microelectron Eng 153:29-36

Krüger-Sehm R, Bacucz P, Jung L, Wilhelms H (2007) Chirp calibration standards for surface measuring instruments. Tech Mess 74:572-576

Leach RK (ed) (2011) Optical measurement of surface topography. Springer, Berlin/Heidelberg

Leach RK, Giusca CL, Haitjema H, Evans C, Jiang $X$ (2015) Calibration and verification of areal surface texture measuring instruments Ann. CIRP 64(2):797-813

Xu ZW, Fang FZ, Gao HF, Zhu YB, Wu W, Weckenmann A (2012) Nano fabrication of star structure for precision metrology developed by focused ion beam direct writing Ann. CIRP 61(1):511-514 